\title{
Histoire du monde indien, 1984-201
}

\section{Gérard Fussman}

\section{(2) OpenEdition}

Journals

Édition électronique

URL : https://journals.openedition.org/annuaire-cdf/1074

DOI : 10.4000/annuaire-cdf. 1074

ISBN : 978-2-7226-0325-7

ISSN : 2109-9227

Éditeur

Collège de France

Édition imprimée

Date de publication : 1 avril 2013

Pagination : 792-794

ISBN : 978-2-7226-0198-7

ISSN : 0069-5580

Référence électronique

Gérard Fussman, « Histoire du monde indien, 1984-201 », L'annuaire du Collège de France [En ligne],

112 | 2013, mis en ligne le 22 novembre 2013, consulté le 22 août 2022. URL : http://

journals.openedition.org/annuaire-cdf/1074 ; DOI : https://doi.org/10.4000/annuaire-cdf.1074 
CR de Robert Calasso, La Folie Baudelaire, trad. de l'italien, Paris, Gallimard, 2011, \& de Yves Bonnefoy, Sous le signe de Baudelaire, Paris, Gallimard, 2011, «D'un Baudelaire l'autre: Yves Bonnefoy et Roberto Calasso », Commentaire, ${ }^{\circ}$ 138, 2012, 587-590.

\section{Préfaces}

- de Guillaume Métayer, Nietzsche et Voltaire, Paris, Flammarion, 2011, 7/9.

- de «Notes pour un portrait», Poètes, princes et collectionneurs, Mélanges offerts à Jean-Paul Barbier-Mueller, Genève, Librairie Droz, 2011.

- de Alexandre Maral, Le Roi-Soleil et Dieu, Paris, Perrin, 2012.

- de Bernard Hoerni, Une éducation en terre berbères, 1940-1958. L'olive, le doum et l'orange, Anet, Atelier Fol'fer, 2012.

- de Marcel Vicaire, Souvenirs du Maroc. Un peintre au Maroc de 1922 à 1958. Dans le sillage de Lyautey, Casablanca, Éditions Afrique-Orient, 2012.

\section{Entretien}

«La métaphore est la clef de la mémoire de la langue », avec Gilles Noussenbaum, Revue décision Santé, $\mathrm{n}^{\circ}$ 284, 2012, 34-35.

\section{Interview}

« Rien de ce qui est dit en français ne m'est étranger », de Sébastien Lapaque, Le Figaro, 19 avril 2012, 8.

«Ce sensualisme de la parole que nous avons perdu », de Jean-Paul Enthoven, Le Point, 22 mars 2012, n 2062, 98-99.

«Les Lumières et l'art sacré », du frère Philippe Markiewicz, Arts sacrés, n 8, 2010, 28-35.

\section{Gérard FussmaN}

\section{Histoire du monde indien, 1984-2011}

\section{ACTIVITÉS EN 2011-2012}

Le professeur, bien qu'à la retraite, a pu continuer à se cultiver et à écrire. Il collabore également au projet de numérisation et de mise sur internet des archives du Collège de France. La mise sur internet des 20000 documents photographiques numérisés de l'Institut d'Études Indiennes a été ralentie par le choix d'un nouveau logiciel (Pleade au lieu de Portfolio) qui exige une nouvelle hiérarchisation des données et une rédaction plus détaillée des légendes, celles-ci servant de base à l'indexation. Le professeur, aidé en cela par Éric Ollivier, y a consacré une partie de son temps, et continuera en 2012-2013.

Le professeur a abandonné en juillet 2012 la présidence de la SEECHAC au profit de Frantz Grenet. 


\section{PuBLICATIONS}

Fussman G. et Quagliotti A.M., The Early Iconography of Avalokiteśvara, Paris, Collège de France, Publications de l'Institut de Civilisation Indienne, fasc. 80, 2012, 152 p.

«Entretien avec Jean-François Sabouret» (sur la formation et la carrière scientifique du professeur avant son élection au Collège de France et sur la façon dont celle-ci s'est déroulée), DVD réalisé par Jean-François Sabouret et Momoko Seto, Collection Paroles d'Asie et du Pacifique, Réseau Asie-Imasie (CNRS), 2011, www.reseau-asie.com.

Fussman G., «The Riddle of the Ancient India eras is not yet solved », Ancient India, Bulletin of the Archaeological Survey of India, New Series, 1, 2011, 239-259.

Fussman G., «Qu'y a-t-il dans un nom: Imrā au Nouristan », dans Azarnouche S. et Redard C. (éd.), Yama/Yima, Variations indo-iraniennes sur la geste mythique, Paris, Collège de France, Publications de l'Institut de Civilisation Indienne, fasc. 81, 2012, 67-82.

Fussman G., «Cours : Le Gandhāra, terre de passage, d'échanges et de création. Séminaire : Étude de documents en rapport avec le cours et leçon terminale », Annuaire du Collège de France, 2010-2011, 489-499.

Fussman G., «Georges Redard, 1922-2005 », nécrologie en anglais dans Encyclopaedia Iranica on line.

Compte rendu du livre de Glass A. (avec une contribution de Mark Allon), Four Gāndhāa $\bar{\imath}$ Samyuktāgama Sūtras, Senior Kharoșthī Fragment 5, Gandhāran Buddhist Texts, vol. 4, Seattle, University of Washington Press, 2007, XXIII + 252 p., dans Indo-Iranian Journal, 55, 2012, 171-200.

\section{CONFÉRENCES}

12 octobre 2011, Rome, Museo Nazionale d'Arte Orientale: «The beginnings of the iconography of Avalokiteśvara in India ».

29 octobre 2011, Université de Caboul : «Le bouddhisme ancien ».

30 octobre 2011, camp des archéologues de Mes Aynak : «Les monastères bouddhiques ».

2 novembre 2011, Université de Caboul : «La personne du/des Buddha-s ».

30 novembre 2011, Paris, La Documentation française : participation au débat sur la situation en Afghanistan.

20 mai 2012, camp des archéologues de Mes Aynak (Afghanistan) : «Les monuments bouddhiques de la région de Caboul ».

22 mai 2012, Université de Caboul, journée d'études organisée par la DAFA et le département d'archéologie de l'université, «Les monuments bouddhiques de la région de Caboul».

\section{MISSIONS}

10-14 octobre 2011, Rome: présidence du $2^{\mathrm{e}}$ colloque international de la Societé européenne pour l'étude des civilisations de l'Himalaya et de de l'Asie Centrale (SEECHAC). Table ronde sur Avalokiteśvara en Inde et Asie centrale au Museo Nazionale d'Arte Orientale.

26 octobre -3 novembre 2011 : mission à Caboul, en compagnie d'Éric Ollivier, sous les auspices de la Délégation archéologique française en Afghanistan, pour étude des monuments bouddhiques en cours de fouille (Tepe Narenj et Mes Aynak) et conférences à l'université. 
12 au 26 mai 2012 : mission à Caboul en compagnie d'Éric Ollivier, sous les auspices de la Délégation archéologique française en Afghanistan, pour étude des monuments bouddhiques en cours de fouille (Tepe Narenj et Mes Aynak) et conférences à l'université.

M. Jacques GERNET, membre de l'Institut

(Académie des inscriptions et belles-lettres)

Histoire sociale et intellectuelle de la Chine, 1975-1992

\section{PUBLICATIONS}

Gernet J., Die Begegnung Chinas mit dem Christentum, Monumenta Serica, Monograph Series XLII, Sankt Augustin, 2012, 413 p.

Gernet J., « Astronomie et calendrier. Sur un livre récent de J.-C. Martzloff », T'oung Pao, Revue internationale de sinologie, vol. XCVII, fasc. 4-5, 2011, 371-392.

M. Jean GUILAINE, membre de l'Institut

(Académie des inscriptions et belles-lettres)

\section{Civilisations de l'Europe au Néolithique et à l'Âge du bronze, 1994-2007}

\section{PuBlicATIONS}

\section{Ouvrages}

Guilaine J., Archéologie, science humaine, entretiens avec Anne Lehoërff, Actes-Sud/ Errance, 2011, 240 p.

Guilaine J., Méditerranée mégalithique. Dolmens, hypogées, sanctuaires, LacapelleMarival, Éditions Archéologie nouvelle, Col. «Archéologie vivante », 2011, 157 p., 217 fig.

\section{Articles}

Guilaine J., «Le Prédynastique vu de la Méditerranée », Archéo-Nil, 20, décembre 2010, Cybèle, 17-24.

Guilaine J., «Megalitos de Francia : distribución geográfica y cronologia / Megaliths in France: geographical distribution and chronology » in Scarre C., Garcia-Sanjuan L. et Weatley D.-W. (dir.), Exploring time and matter in prehistoric monuments: debating absolute chronology and rare rocks in European megaliths, Menga, Serie Monografica, 01, Antequera, 2011, 76-101. 